\title{
Manuka honey a valuable addition to the wound dressing
} \section{armamentarium}

dos Santos, BP*\$‡; Serracanta Domenech, $\mathrm{J}^{*}$; Aguilera Saez, $\mathrm{J}^{*}$; Bosacoma Roura, $\mathrm{P}^{\star} \S$; Barret Nerin, JP ${ }^{*} \dagger$

* Vall d'Hebron University Hospital, Barcelona, Spain

¥ Corresponding author

§ Plastic surgery resident

${ }^{\dagger}$ Plastic surgery consultant

tHead of plastic surgery department
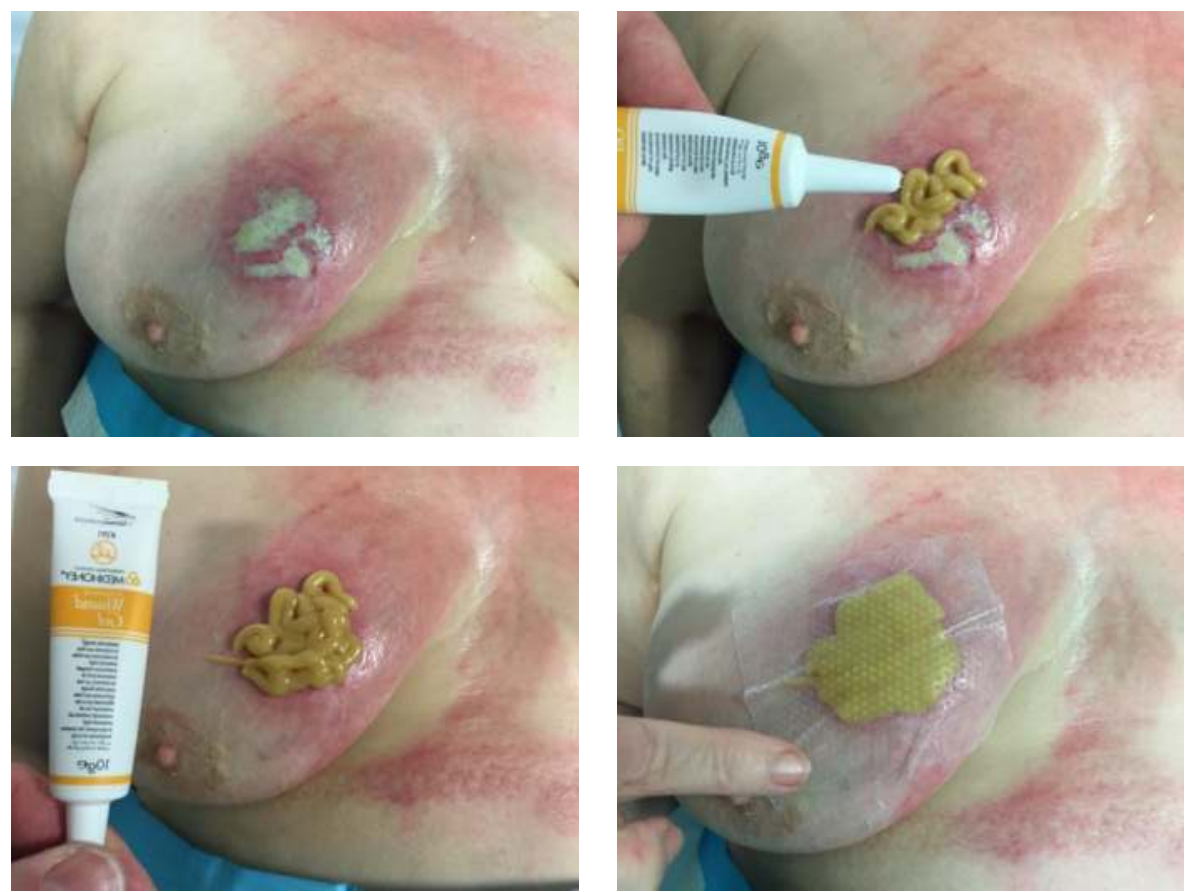

Figure 1. Manuka honey dressing

\section{Objectives}

To outline our experience with Manuka honey at our regional burn center, as an advance wound therapy for complex or stagnant wounds

\section{Methods}

We describe the current recommendations made by the manufacturer and outline in the relevant literature.

We also summarize our experience with complex and stagnant wounds in the burn patient population. The treatment protocol used at our center consists in applying commercially available Manuka honey paste and then a commercially available impermeable wound contact layer and finally covered up with a dry sterile gauze, this dressing was changed once every three days with alternate treatments either at home or a local healthcare facility
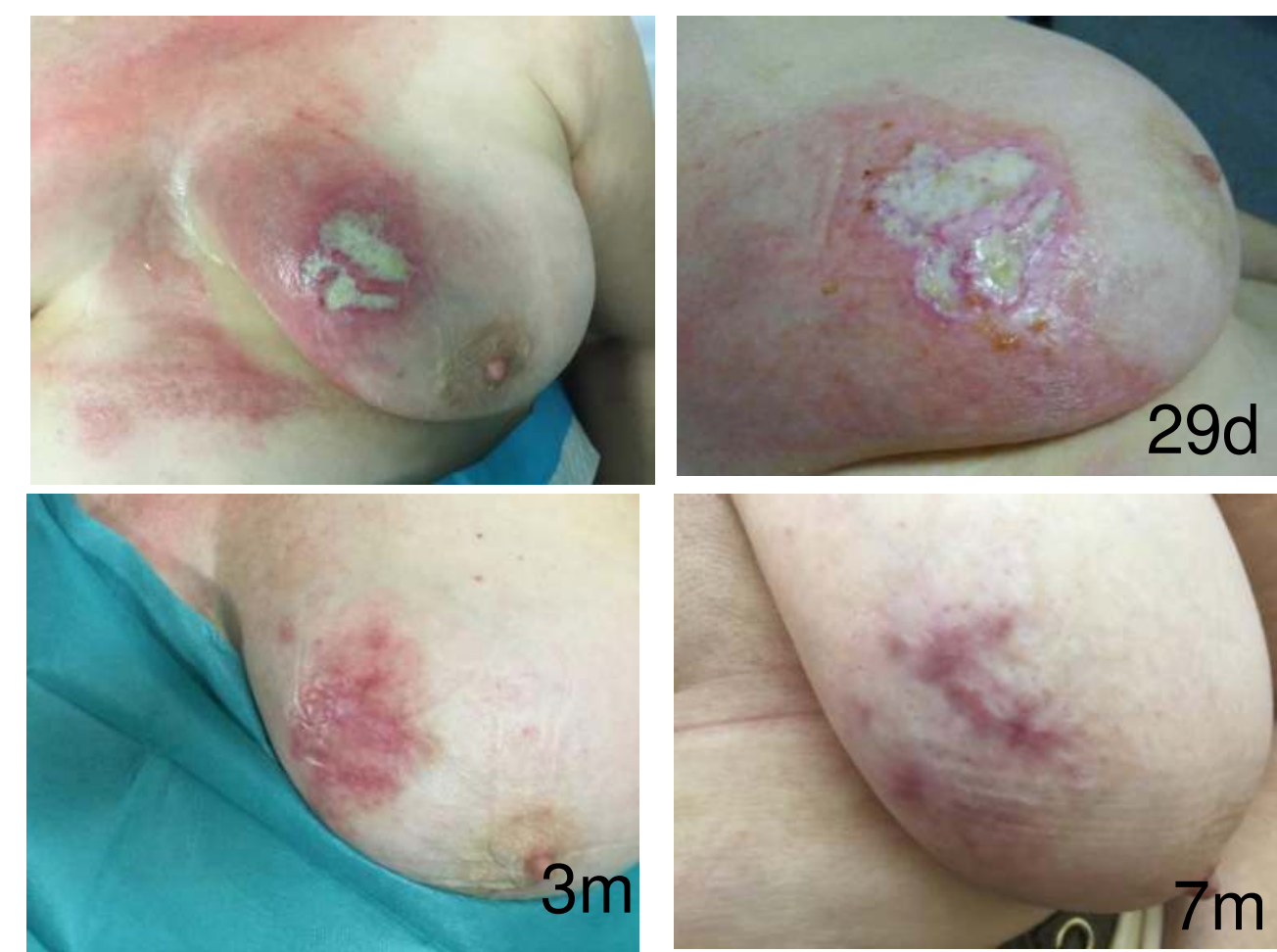

Figure 2. Burn victim at presentation and follow up
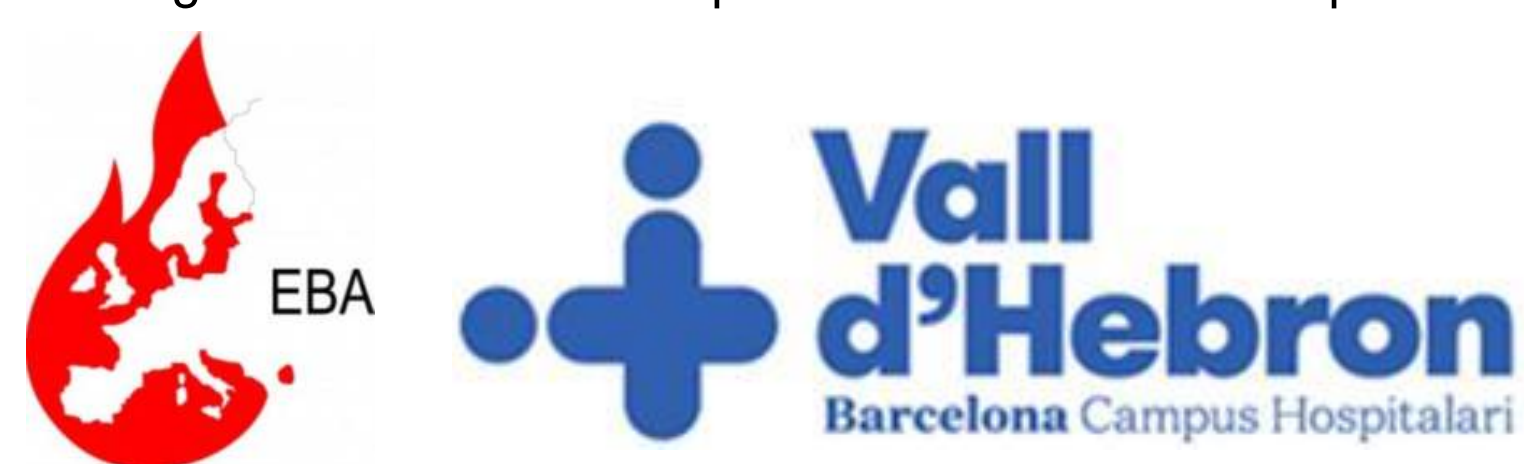

Barcelona Campus Hospitalari
Manufacturer's directions

Apply a 3mm layer

Apply directly or use a secondary dressing

Based on the level of exudate change in up to 7 days

Honey does not cause maceration

Cautions

Honey allergy

What we do (every 3 days):

Apply Medihoney ${ }^{\circledR}$ paste directly over the wound bed

Cover with a sheet of Mepitel-one ${ }^{\circledR}$

Dry gauze dressing

\section{Results}

Manuka honey is a valuable complement to the available therapies and strategies used to manage recalcitrant wounds in burn patients

12 patients were successfully treated during the last year.

Our center's Manuka honey protocol allows for outpatient treatment and follow-up with the possibility of scheduling alternate visits at the local health center unburdening our facility.

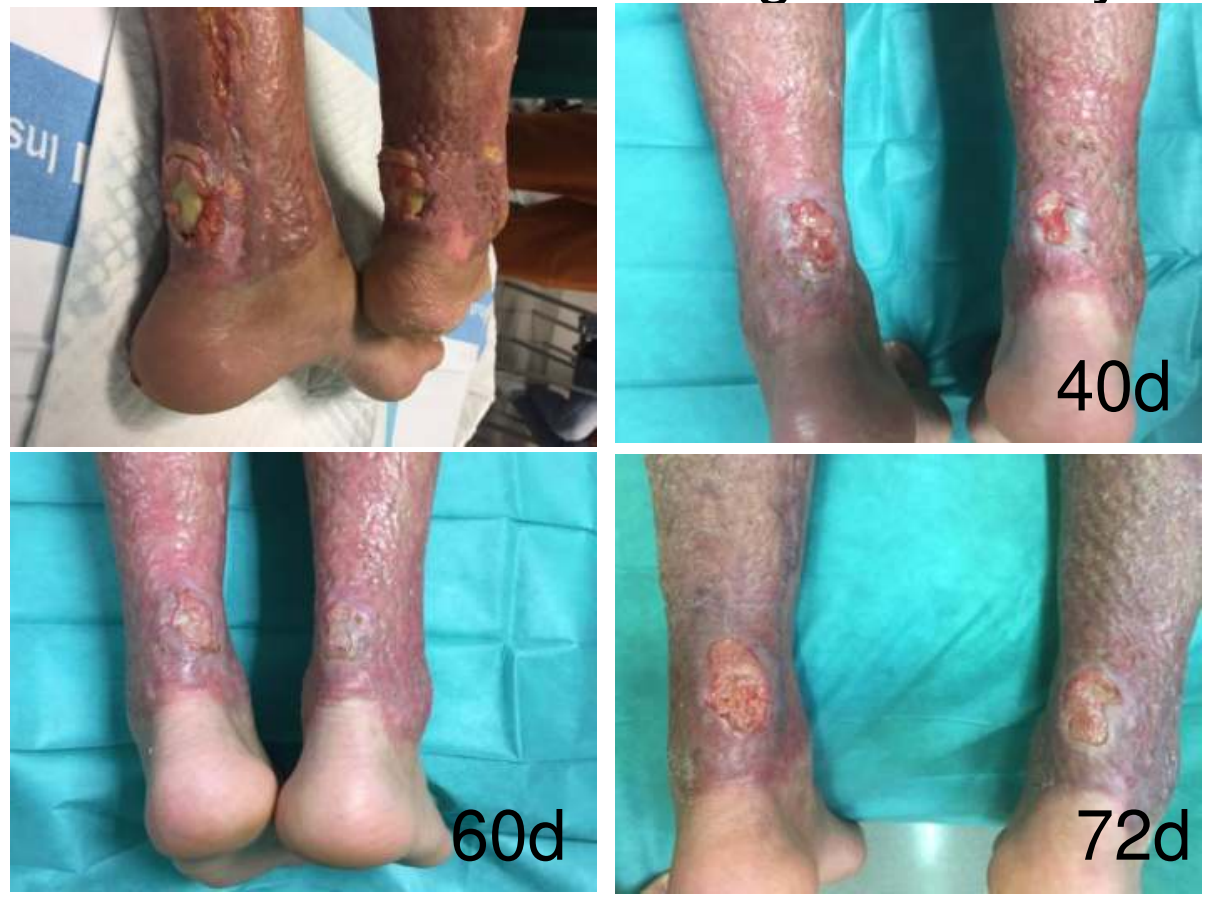

Figure 3. Treatment of burn victim with chronic wound

\section{Discussion / Conclusion}

The presence of conditions that impose an insufficient wound bed microvascular-wise represents a difficult challenge where both aggressive and conservative approaches proved inadequate or at least untimely. In this subset of patient population, Manuka honey proved an effective strategy to restart the wound healing process albeit some cases still remain challenging.
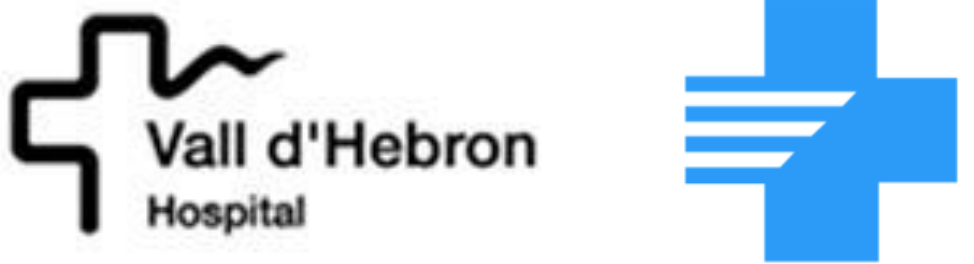

Institut Català de la Salut 